\title{
Effectiveness of Highly Active Anti-retroviral Therapy (HAART) in the Adult Population in the Ashanti Region of Ghana
}

\author{
Kwaku G. Oppong ${ }^{1}$, Eric Boakye-Gyasi², Kwesi B. Mensah'², Ralph Obeng ${ }^{3}$ \\ Akua A. Abruquah ${ }^{4}$, Eric Woode ${ }^{5}$
}

\author{
${ }^{1}$ Department of Pharmacology, Faculty of Pharmacy and Pharmaceutical Sciences, \\ College of Health Sciences, Kwame Nkrumah University of Science \& Technology. Kumasi - Ghana \\ ${ }^{2}$ Department of Child health, Komfo Anokye Teaching Hospital. Kumasi - Ghana \\ ${ }^{3}$ Department of Medicine, Anti-Retroviral Center, \\ Komfo Anokye Teaching Hospital. Kumasi - Ghana \\ ${ }^{4}$ Department of Pharmaceutical Sciences, faculty of Health Sciences, \\ Kumasi Technical University. Kumasi - Ghana \\ ${ }^{5}$ Department of Pharmacology and Toxicology, School of Pharmacy, University of Allied \\ Health Sciences. Ho - Ghana
}

\begin{abstract}
AIDS is the late stage of infection with the Human Immunodeficiency Virus (HIV). Even though the Highly Active Antiretroviral Therapy (HAART) for the management of HIV/ AIDS has been in existence in Ghana since 2003, there is still limited data on their effectiveness. This study therefore sort to ascertain the effectiveness of HAART used at Anti-retroviral therapy (ART) Centres in the Ashanti region of Ghana by assessing its effects on immunological and haematological markers as well as some significant indicators such as body temperature and blood pressure changes. The study was a retrospective cross sectional study which reviewed medical record folders of $156 \mathrm{HIV}$ infected patients who had attended the selected ART centres in the Ashanti region of Ghana between 2010 and 2014 . About $49 \%$ of the study population had a significant rise in their CD4 counts after six months strict adherence to specific HAART regimen. HAART effectively reduced viral loads and increased $\mathrm{CD}_{4}$ counts in most of the patients whose medical record folders were reviewed. Socioeconomic factors, formal education and lack of health literacy had a significant effect on adherence to the HAART regimen, however; marital status of patients did not have any significant effect on adherence, $\mathrm{CD}_{4}$ counts and the haemoglobin concentration of patients. Based on results obtained from this study, HAART could be considered as effective in the adult population in the Ashanti region of Ghana provided there is a high level of adherence. Immunological and haematological markers for the assessment of effectiveness could also improve with high adherence recommended HAART regimen.
\end{abstract}

Keywords: CD4, HAART, haematological, immunological, viral load

Corresponding author: Eric Boakye-Gyasi. Departement of Pharmacology, Faculty of Pharmacy and Pharmaceutical Sciences, Kwame Nkrumah University of Science \& Technology. Kumasi, Ghana. Email: ebgyasi.pharm@knust.edu.gh Received: 20 January 2021. Revised: 7 April 2021. Published: 5 August 2021 


\section{Introduction}

Acquired Immune Deficiency Syndrome (AIDS) is an infection with the human immunodeficiency virus (HIV) leading to a total breakdown of the immune system of the host which results in a number of opportunistic infections. ${ }^{1}$ The damage to the host's immune system also results in a number of immunological and haematological complications.

In recent years, stakeholders in the health industry all over the world have committed a lot of resources to fight the HIV/AIDS pandemic and to prevent its spread. Although progress has been made in the campaign against HIV, over 2 million new HIV infections occurred in 2009 with almost 2 million AIDS related deaths. ${ }^{2}$ In 2012, the prevalence of HIV was $1.37 \%$ in Ghana. A total of about 235,982 persons were living with HIV and AIDS. At the end of 2016, HIV prevalence in Ghana was $2.4 \%{ }^{3}$

Sub-Saharan Africa remains the worst affected region in the world. Limited resources have been a major challenge in the fight against HIV/AIDS. In Ghana, AIDS is considered not only as a health problem but one affecting the socioeconomic aspects of life as well. ${ }^{4}$ People living with HIV/AIDS (PLHIV) need effective care. This could be achieved with the use of the highly active antiretroviral therapy (HAART) in patients who satisfy the requirements of its usage. Decision to start ART was based on clinical and immunological outcomes, ${ }^{5}$ but now the current policy recommends that, all HIV infected individuals should be on HAART. ${ }^{6}$

HAART is a combination of three or four antiretroviral medicines aimed at reducing the risk of drug resistance. It has been shown in countries where HAART is used that, mortality and morbidity rates have waned considerably. The pivotal role of HAART is to reduce the replication of the virus and to give the individual's immune system the opportunity to recover. This prevents the progression to AIDS. ${ }^{2}$ The main effect of HIV infection is destruction of CD4 T-lymphocytes through a number of mechanisms which include HIV induced cytolysis, dysregulation of cytokines among others. $^{2}$ Haematological complications including anaemia, neutropoenia, lymphopoenia and thrombocytopenia have been demonstrated to be the second highest cause of morbidity and mortality in HIV patients. $^{5}$

Highly active HAART has been globally accepted as the main stay of medicines for the management of HIV patients. There are however conflicting reports on the efficacy and the capability of HAART to better the quality of life of HIV patients.

The main objective of the study was to ascertain the effectiveness of HAART on markers ofHIV disease progression, morbidity and mortality in the adult population of HIV/ AIDS patients in the Ashanti Region of Ghana as a way of assessing its effectiveness.

\section{Methods}

Design of Study

The study was a retrospective cross sectional survey. It involved the use of data-collecting tools to review patient medical record folders at the Anti-retroviral therapy (ART) centres in three health facilities in the Ashanti Region of Ghana. Medical record folders of patients who attended ART clinic from 2010 to 2014 were reviewed. A period of four months was used in the data collection.

\section{Ethical Considerations}

The study had the approval of the Komfo Anokye Teaching hospital, the Ghana 
Health Service and the Committee on Human Research, Publication and Ethics (CHRPE) (Reg. No. RD/CR14/174) at the Kwame Nkrumah University of Science and Technology. Data was entered into a passworded computer by the principal investigator to ensure the safety of patient information.

\section{Study Sites}

The study sites were Komfo Anokye Teaching Hospital (KATH), Obuasi Municipal Hospital, and Asante Mampong Municipal Hospital. KATH is located in Kumasi, the regional capital of Ashanti Region of Ghana and it is the major referral centre for the Ashanti, Brong Ahafo, parts of Western, Central and the Northern Region of Ghana.

Obuasi Municipal hospital is the largest district hospital located in the southern part of the Ashanti Region. The hospital is located in Obuasi, a town noted for its gold and other natural resources. This attracts people from all over the country to engage in trade.

Asante Mampong Municipal hospital is located in Asante Mampong which is in the northern part of the Ashanti region. The town is well known for its numerous educational institutions including a university, teacher training colleges, nursing and midwifery training colleges, a number of senior high schools and thus bringing together people from different parts of the country. The hospital serve patients from many parts of the country, more especially the northern part of Ashanti region, the north-eastern part of the Brong Ahafo region and the western part of the Eastern region.

\section{Study Population and Sampling Technique}

According to the health administrators of the three facilities, the average number of HIV/ AIDS patients attending the ART centre in a week for all three facilities was 170 patients. The sample determination formula ${ }^{7}$ below was used to determine the study participants. $\mathrm{N}$ is the sample frame (170), $\mathrm{n}$ is the sample size and e is the margin of error (level of precision)

$$
n=\frac{N}{1+\left(N e^{2}\right)}
$$

Where $\mathrm{N}=170$ and $\mathrm{e}=5 \%$

$$
\begin{gathered}
n=\frac{170}{1+\left(170 * 0.05^{2}\right)} \\
n=119.29 \\
n \approx 119
\end{gathered}
$$

Thus with a margin of error of 0.05 (that is confidence interval of 95\%), 119.29 (approximately, 119) HIV/AIDS patient medical record folders would be reviewed. The probability proportional sampling technique was used to determine the number of HIV/AIDS patient's medical record folders to be reviewed from each of the health facilities. Patient medical record folders were randomly selected and reviewed with data collecting tools designed by the investigators.

Inclusion and Exclusion Criteria

Patient medical record folders which satisfied the following requirements were included in the study

1. Patients who have been on HAART for a minimum of six months

2. Patients who were 13 years and above

3. Patients who had attended ART centres between 2010 and 2014.

The following groups of patients were not included in the study.

1. Pregnant women

2. Children below the age of 13

3. Patients on antitubercular drugs

Data Handling and Analysis

A total of 170 patient medical record folders were reviewed. The key markers monitored 
Table 1. Age and Gender of Patients

\begin{tabular}{ccccc}
\hline Age Range (years) & Male & Female & Total & Cumulative (\%) \\
\hline $14-30$ & 4 & 28 & 32 & 20.5 \\
$31-50$ & 29 & 65 & 94 & 60.2 \\
$51-70$ & 10 & 17 & 27 & 17.3 \\
Above 70 & 0 & 3 & 3 & 1.9 \\
Total & $43(27.6 \%)$ & $113(72.4 \%)$ & 156 & 100 \\
\hline
\end{tabular}

Source: Survey Data, 2015

included the $\mathrm{CD}_{4}$ counts, viral loads, haemoglobin $(\mathrm{Hb})$, blood pressure, body temperature and weight of patients. Data were analysed with Statistical Package for Social Sciences (SPSS) version 21. The results were analysed with a 95\% Confidence Interval ( $\mathrm{P}<$ 0.05). The Spearman Rank Order Correlation was performed to assess the strength and direction of the relationship on the effect of HAART on haemoglobin and $\mathrm{CD}_{4}$ counts. It was also used to assess the HAART combination that had significant effect on the markers such as $\mathrm{CD}_{4}$, haemoglobin, blood pressure and body temperature. The null hypothesis was that the HAART may not have effect on $\mathrm{CD}_{4}$ counts, viral loads and haemoglobin of patients. This decision rule will be rejected at a significant value of $p$ equal to or less than 0.005 .

\section{Results and Discussion}

\section{Demographic Characteristics of Patients}

Results from the study indicated that majority of the patients $(60.2 \%)$ were in the age groupings of 31-50 years and most of the patients $(72.4 \%)$ were females (Table 1$)$. The mean age group of patients attending ART clinics in the Ashanti region between 2010 and 2014 was $31-50$ years. This is consistent with other studies, which indicated that the incidence of HIV/AIDs in the world is highest with the ages between 21-49 years. ${ }^{8-10}$ This result also reflects the national prevalence of HIV/AIDS in Ghana, which is highest with the ages of $45-49$ years $(5.6 \%)$, followed by
35-39 years (3.5\%), and then 15-19 years $(0.6 \%) .{ }^{3}$ Majority of the study population were also females $(72.4 \%)$.

In 2004, the Ghana AIDS commission estimated the prevalence of HIV/AIDS among females of 15 years and above to be about $60.2 \%$. $^{3}$ The study results therefore reflect the national prevalence of $\mathrm{HIV} /$ AIDS in Ghana. However, these results could also imply that the prevalence of HIV/ AIDS in the Ashanti region may be higher among the female population. A study in five African countries observed that among most discordant couples, majority of the infected partners were females. ${ }^{11}$

As depicted in Table 2 below, more than half $(59.6 \%)$ of the patients whose medical record folders were reviewed were married. This means that majority of them had legal and socially accepted sexual partners. A high prevalence of HIV/AIDS among married couples was also observed in the study. This high prevalence of HIV infection among married respondents may also be indicative of multiple sexual partners and the practice of unsafe sex among majority of the couples in this study. This outcome is also consistent with literature. The World Health Organization (WHO) stated in its policy on treatment and management of HIV in 2015 that the reason for the spread of HIV among married couples was mostly due to unprotected or unsafe sex as well as multiple sexual partners. ${ }^{1}$ 
Table 2. Marital Status, Educational Level and Occupational Status of Patients

\begin{tabular}{llc}
\hline \multicolumn{2}{c}{ Demographic Characteristics } & Frequency n (\%) \\
\hline Marrital Status & Single & $20(12.8)$ \\
& Married & $93(59.6)$ \\
& Divorced & $28(17.9)$ \\
& Widow/Widower & $11(7.1)$ \\
& Separated & $4(2.6)$ \\
Education Level & Uneducated & $30(19.2)$ \\
& Primary & $29(18.6)$ \\
& Middle School & $31(19.9)$ \\
& Junior High School & $41(26.3)$ \\
& High School & $17(10.9)$ \\
& Tertiary & $8(5.1)$ \\
Occupation & Farmer & $23(14.7)$ \\
& Trader & $95(60.9)$ \\
& Teacher & $12(7.7)$ \\
& Health Worker & $1(0.6)$ \\
& Unemployed & $15(9.6)$ \\
& Studed & $6(3.9)$ \\
& Laborer & $1(0.6)$ \\
& Office Work (Secretary) & $3(1.9)$ \\
\hline
\end{tabular}

Source: Survey Data, 2015

With regards to education, about $84 \%$ of patients had between primary and junior high education. Only about $16 \%$ had between senior high and tertiary education. It is evident that, majority (58\%) of the patients had low level educational background with approximately $19 \%$ stated as not having received any form of formal education. The level of education of patients could indirectly influence the effectiveness of HAART. Some studies indicate that, the number of years of formal education was found to be independently associated with virologic success among HIV/AIDS patients. The educational level of a patient as well as his or her level in health literacy has effect on the patient's attitude towards adhering to medication regimen. ${ }^{12}$
In addition, the educational background of individuals has influence on their attitude to curb the spread of a disease, their ability to understand how infections occur and the need for the HAART to be taken for life. Highly educated individuals are better educated to understand the mode of transmission of a disease and how to manage it. This in turn affects families, communities and the society as a whole. ${ }^{13}$ Other studies have observed the importance of the incorporation of the education on HIV/AIDS into main stream academic curricula. It has been found to be a key measure in curbing the spread of the disease. ${ }^{14}$

Table 2 also shows that majority of patients $(60.9 \%)$ were engaged in trading. This was followed by about $15 \%$ being farmers, and 
Table 3. Effect of HAART on Markers for Assessing HIV Patients

\begin{tabular}{lll}
\hline \multicolumn{1}{c}{ Markers for Assesments } & Frequency n (\%) \\
\hline $\mathrm{CD}_{4}$ count $($ cells/mL) & Above 350 & $80(51.3)$ \\
& Below 350 & $60(38.5)$ \\
& No Record & $16(10.2)$ \\
$\mathrm{Hb}$ Concentration $(\mathrm{g} / \mathrm{dL})$ & Below 12.6 & $81(51.9)$ \\
& Above 12.6 & $35(22.5)$ \\
& No Record & $40(25.6)$ \\
Viral Load (cells/mL) & $501-1000$ & $1(0.6)$ \\
& $1001-1500$ & $1(0.6)$ \\
& Above 10000 & $1(0.6)$ \\
Temperature $\left({ }^{\circ} \mathrm{C}\right)$ & No Record & $153(98.1)$ \\
& Below 37 & $109(69.9)$ \\
& Above 37 & $7(4.5)$ \\
Blood Pressure $(\mathrm{mm} / \mathrm{Hg})$ & No Record & $40(25.6)$ \\
& Below 120/80 & $30(19.2)$ \\
& Above $120 / 80$ & $7(4.5)$ \\
& Exactly $120 / 80$ & $82(52.6)$ \\
& No Record & $37(23.7)$ \\
\hline
\end{tabular}

Source: Survey Data, 2015

$9.6 \%$ being unemployed. The high prevalence of HIV among traders in this study confirms the WHO report on HIV/AIDS in 2015, which considered traders and long distance drivers as groups at high risk of getting infected with and spreading the HIV. Some traders travel long distances and for over a long period are away from their spouses or regular sexual partners. They are more likely to meet many other persons and may have unsafe sex in the cause of trading and travelling around. ${ }^{15}$ Occupational status is a cardinal factor that determines the socioeconomic status of an individual. This may influence the nature of care for HIV/AIDS persons.

Effect of HAART on markers for assessing HIV Patients

The effectiveness of HAART may be evident in the laboratory results of $\mathrm{CD}_{4}$ counts and viral loads. As shown in Table 3, a total of about $38 \%$ of patients had their average $\mathrm{CD}_{4}$ count above 350 cells $/ \mathrm{mL}$ after being on the HAART for six months. However, about 51\% had $\mathrm{CD}_{4}$ counts below the recommended 350 cells $/ \mathrm{mL}$ after six months of being on HAART. The study showed that there were no records on viral loads for over $98 \%$ of patients.

WHO recommended that HAART should be started on patients whose $\mathrm{CD}_{4}$ counts fall below 350 cells $/ \mathrm{mL}$. This was the guideline that was used by the Ministry of Health in Ghana during the period of this study. With this reference, about $38 \%$ of patients had their average $\mathrm{CD}_{4}$ counts above the critical line set by the WHO. This was after these patients had taken HAART for a minimum of six months. It was also observed that, about $51.3 \%$ of patients had their average $\mathrm{CD}_{4}$ counts below 350 cells $/ \mathrm{mL}$ after at least 


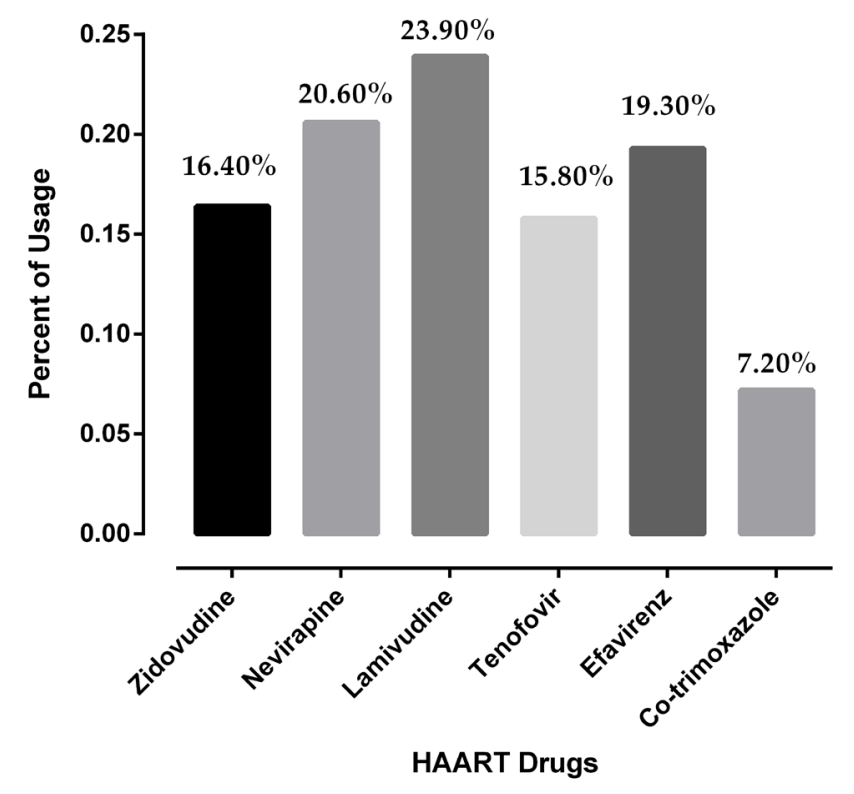

Figure 1. ARVs Dispensed to Patients in the Three Health Facilities in the Ashanti Region

six months on HAART. The reason for this may be varied. Non-adherence to HAART dosage regimen could be a major factor which could deny patients of the full benefit of the therapy. Secondly, there is evidence that genetic variations in drug metabolism, early drug resistance and concurrent opportunistic infections could also influence the effectiveness of HAART. ${ }^{16}$

The results also indicated that about $51.9 \%$ of the patients had their haemoglobin $(\mathrm{Hb})$ concentration below $12.6 \mathrm{~g} / \mathrm{dL}$ with approximately $22.5 \%$ of patients having $\mathrm{Hb}$ concentrations above it. However, about $25.6 \%$ of patients had no records of their $\mathrm{Hb}$ concentrations in their medical record folders as indicated in Table 3 . The optimal $\mathrm{Hb}$ concentration for males is $13.8-17.2 \mathrm{~g} / \mathrm{dL}$ and $12-15.6 \mathrm{~g} / \mathrm{dL}$ for females. The majority $(51.9 \%)$ of patients' $\mathrm{Hb}$ level indicated they were anaemic. This may be due to a number of reasons. In the first place, it may be that the HAART may not have been effective because patients reported of high compliance of $89 \%$.
There were records in only two patient medical record folders on viral load. A viral load less than 10,000 copies per $\mathrm{ml}$ is generally accepted globally for HIV/AIDS patients. From Table 3, it was realized that the temperature of about $70 \%$ of patients from all the three health facilities were below $37^{\circ} \mathrm{C}$. The temperatures of the remaining $5 \%$ of the patients were above $37^{\circ} \mathrm{C}$. Increase in body temperature is one indication of infection and in the case of HIV/AIDS patients; they are more prone to opportunistic infections due to compromised immune systems.

The study further indicated that more than half $(52.6 \%)$ of the patients had their blood pressure at $120 / 80 \mathrm{mmHg}$ as shown in Table 3 below. However, about $20 \%$ had their blood pressure below 120/80 $\mathrm{mmHg}$. The remaining $4 \%$ had blood pressure readings above $120 / 80 \mathrm{mmHg}$. There were no records of blood pressure readings for $24 \%$ of patients. A spearman correlation analysis showed a significant relationship between the ages of patients and the changes in the $\mathrm{CD}_{4}$ 
Table 4. Effect of HAART on $\mathrm{CD}_{4}$ Count, Haemoglobin Concentration, Body Temperature, and Blood Pressure

\begin{tabular}{cccccc}
\hline \multicolumn{2}{c}{$\begin{array}{c}\text { HAART } \\
\text { Combination }\end{array}$} & CD $_{4}$ Count & $\begin{array}{c}\text { Haemoglobin } \\
\text { Concentration }\end{array}$ & $\begin{array}{c}\text { Body } \\
\text { Temperature }\end{array}$ & $\begin{array}{c}\text { Blood } \\
\text { Pressure }\end{array}$ \\
\hline Zidovudine, & Correlation & $0.285^{* *}$ & $0.246^{* *}$ & $0.660^{* *}$ & -0.088 \\
Lamivudine, & coefficient $\alpha$ & 0.001 & 0.008 & 0.000 & 0.288 \\
Efavirenz & value N & 135 & 114 & 148 & 147 \\
Zidovudine, & Correlation & 0.149 & $0.254^{* *}$ & $0.453^{* *}$ & -0.148 \\
Lamivudine, & coefficient $\alpha$ & 0.084 & 0.006 & 0.000 & 0.074 \\
Neviraprine & value N & 135 & 114 & 148 & 147 \\
Zidovudine, & Correlation & $0.285^{* *}$ & $0.252^{* *}$ & $-0.380^{* *}$ & 0.027 \\
Lamivudine & coefficient $\alpha$ & 0.001 & 0.007 & 0.000 & 0.748 \\
& value N & 135 & 114 & 149 & 147 \\
\hline
\end{tabular}

$* *$. Correlation significant at the 0.01 level (2-tailed)

counts $(\mathrm{p}=0.001)$ with a positive correlation coefficient of 0.289 .

\section{Specific ART Dispensed to Patients}

Many different antiretrovirals were dispensed to patients. These included a combination of the following; zidovudine + lamivudine + nevirapine, or zidovudine + lamivudine + efavirenz or zidovudine + tenofovir + efavirenz or zidovudine and lamivudine alone. Lamivudine was the most frequently used ART in the combination for patients. It constituted about 24\%. This was followed by nevirapine $(20.6 \%)$, efavirenz, (19.3\%), zidovudine (16.4\%) and tenofovir (15.8\%) as shown in Figure 1. Analysis of the results indicated that the zidovudine + lamivudine + efavirenz combination increases $\mathrm{CD}_{4}$ counts better than zidovudine + lamivudine + nevirapine combination. Patients who had been administered with dual therapy of zidovudine and lamivudine alone during periods of shortages of HAART also had significant increase in their $\mathrm{CD}_{4}$ counts better than the combination of zidovudine + lamivudine + nevirapine.
The results as indicated in Table 4 showed that the combination of zidovudine, lamivudine and efavirenz could significantly increase $\mathrm{CD}_{4}$ counts, increase $\mathrm{Hb}$ concentration and reduce raised body temperature. The combination did not have much effect on the blood pressure of patients. In addition, the significant effect of the combination was more on temperature than on $\mathrm{CD}_{4}$ counts and $\mathrm{Hb}$. Also, the results showed a statistical significant effect of the combination of zidovudine, lamivudine and neviraprine on haemoglobin and temperature. However, there was not much effect of this combination on blood pressure and $\mathrm{CD}_{4}$ counts. In addition, the combinations of zidovudine and lamivudine alone indicated a positive significant relationship between the combination on $\mathrm{CD}_{4}$ and $\mathrm{Hb}$. There was also a negative relationship between the combinations on temperature. However, there was no significant relationship between the combinations of zidovudine and lamivudine and blood pressure of the patients.

The study showed that, the combination of Zidovudine, Lamivudine and Nevirapine caused the most significant negative effect 
on haemoglobin compared to Zidovudine, Lamivudine and Efavirenz or Zidovudine and Lamivudine as shown in Table 4 below. This result agrees with literature. Zidovudine and Nevirapine are well known antiretrovirals with anaemia as major side effect. ${ }^{17}$ In the study, nevirapine was the second most prescribed $(20.6 \%)$ of all ARVs. The high frequency of nevirapine use in the majority of patients may have also contributed to the low haemoglobin content of the many patients.

The study revealed that, the body temperature of about $70 \%$ of patients from all the three health facilities were below $37^{\circ} \mathrm{C}$. The temperatures of about $5 \%$ of the patients were above the $37^{\circ} \mathrm{C}$. The combinations of Zidovudine, Lamivudine and Nevirapine and that of Zidovudine, Lamivudine and Efavirenz significantly contributed to the raised body temperatures. High body temperature may be indicative of infection or inflammation. ${ }^{18}$ As indicated by the results of the study, $70 \%$ of patients had their body temperature within normal range. It may be indicative of the control of the HIV infection and the effectiveness of the HAART to control replication of the HIV.

It was also observed that about $70 \%$ of the patients in the study had blood pressure reading recording around 120/80 $\mathrm{mmHg}$. However, there are documented evidence that, patients with long term use of HAART could be predisposed to renal failure, hypertension and coronary heart disease. ${ }^{19-20}$

Effects of frequency of visits to health facility on $C D_{4}$, heamoglobin, and adherence

As indicated in Table 5 below, patients who attended ART centres monthly had better increase in $\mathrm{CD}_{4}$ counts and haemoglobin content compared to those who did not. There was a positive significant relationship between frequency of visits to the health facility by patients and the increase in the $\mathrm{CD}_{4}$ counts. The significant value of 0.001 and a correlation coefficient of 0.279 imply that the more patients visited the health centres, the more their $\mathrm{CD}_{4}$ counts increased. However, the effect of frequency of visits to ART centres was not so significant on adherence to medication regimen.

\section{Limitations of The Study}

Challenges encountered during the study included inadequate records of vital information of patients in the medical record folders. In addition, information on adverse drug action and reasons why prescribers changed the initial HAART combination to another were not well documented in most cases. Few records of laboratory results on the viral load and $\mathrm{CD}_{4}$ counts of patients were documented in patient medical record folders. In many cases, mortalities were not recorded in the medical record folder by clinicians.

\section{Conclusion}

Findings from this current study are indicative that HAART may be effective in increasing $\mathrm{CD}_{4}$ counts as well as reducing the morbidity and mortality in the study population. Also, gender had no significant influence on the effectiveness of HAART but however, age, formal education and occupation had significant influence on adherence and hence the effectiveness of HAART. Additionally, the combination of Zidovudine, Lamivudine and Efavirenz or Zidovudine and Lamivudine could better increase $\mathrm{CD}_{4}$ counts.

\section{Acknowledgements}

Authors are grateful to the Regional Health Directorate of the Ashanti region, the Obuasi and Mampong District Health Directorates and the Medicine Department of the Komfo Anokye Teaching Hospital for their support during this study. 


\section{Funding}

None

\section{Conflict of Interest}

None Declared

\section{References}

1. World Health Organization Policy on treatment and management of HIV (2015). Available at http://www.who. int/hiv/pub/guidelines/en/, (Assessed on May, 2017)

2. Owiredu WKB, Quaye L, Amidu N, Addai-Mensah O. Prevalence of anaemia and immunological markers among Ghanaian HAART-naïve HIV-patients and those on HAART. African Health Sciences. 2011; 11(1): 2-15

3. Ghana AIDS Commission (2016) HIV Sentinel Survey report for 2016. Available at http://www.ghanaids.gov.gh/gac1/ aids info.php (Assessed on May, 2017)

4. Ghana AIDS Commission (2014) 2014 Status report, Available at http://www. ghanaids.gov.gh/gac1/pubs/2014\%20 STATUS\%20REPORT.pdf, (Assessed on May, 2017)

5. Annison L, Dompreh A. Adu-Sarkodie Y. The immunological response of HIVpositive patients initiating HAART at the Komfo Anokye Teaching hospital, Kumasi, Ghana. Ghana Medical Journal 2013; 47 (4):164-70.

6. Fox, M.P. and Rosen, S. A new cascade of HIV care for the era of "treat all". PLoS Medicine. 2017 14(4): e1002268

7. Yamen T. Statistics, An introductory Analysis. 2nd ed, New York: Harper and Row, 1967

8. Amornkul PN, Vandenhoudt H, Nasokho P, Odhiambo F, Mwaengo D, Hightower A, et al. HIV prevalence and associated risk factors among individuals aged 1334 years in rural western Kenya. PloS One 2009; 4 (7):6470.
9. Glynn JR, Caraël M, Auvert B, Kahindo M, Chege J, Musonda R el al. Why do young women have a much higher prevalence of HIV than young men: A study in Kisumu, Kenya and Ndola, Zambia. AIDS 2001; 15 (4 suppl):S51-60

10. UNAIDS (2002). Prevalent rates of HIV/AIDS among age groups in 2002. Available at http://www. who.int/hiv/topics/mtct/meetings/ NairobiFeb07Operationalguide.pdf, (Assessed on May, 2017)

11. de Walque D. Discordant couples: HIV infection among couples in Burkina Faso, Cameroon, Ghana, Kenya, and Tanzania. World Bank Policy Research Working Paper No. 3956; 2006.

12. Kalichman SC, Ramachandran B, Catz S. Adherence to combination of antiretrovirals in HIV patients of low health literacy. Journal of General Internal Medicine 1999; 14(5): 267-273.

13. UNESCO (2015). HIV/AIDS and Education. Available at h t t p :// un es d oc.unesco.org/ images/0012/001286/128657e.pdf, (Assessed on May, 2017)

14. Kelly MJ. The encounter between HIV/ AIDS and education, University of Zambia, Lusaka, Zambia; 2000

15. KMCC Uganda, Most at Risk Populations - Long Distance Truck Drivers and HIV/AIDS in Uganda: Synthesis of Information and Evidence to Inform the Response. Synthesis Report, 2014

16. Campo JE, Jamjian J, Goulston C. HIV Antiretroviral Drug resistance. Journal of AIDS \& Clinical Research 2012; 5(2); 2155-2161

17. Vaneet A, Reshma SR, Somashekar HS, Narendranath S, Keerthisagar J, Dinaka KR. Evaluation of the effect of the antiretroviral regimen (Zidovudine + Lamivudine + Nevirapine) on CD4 count, body weight and $\mathrm{Hb} \%$ on HIV 
patients-a retrospective study. Journal of Interdisciplinary and Multidisciplinary Research 2015; 2 (5):177-185.

18. González Plaza JJ, Hulak N, Zhumadilov Z, Akilzhanova, A. Fever as an important resource for infectious diseases research. Intractable \& Rare Diseases Research 2016; 5(2), 97-102.

19. Palacios J, Santos J, Garcia A, Castells E, Gonzalez M, Ruiz J.et al. Impact of highly active antiretroviral therapy on blood pressure in HIV-infected patients. A prospective study in a cohort of naive patients. HIV Medicine 2006; 7(1):10-15.

20. Röling J, Schmid H, Fischereder M, Draenert R, Goebel FD. HIV-Associated Renal Diseases and Highly Active Antiretroviral Therapy-Induced Nephropathy. Clinical Infectious Diseases 2006; 42(10):1488-1495 